\title{
Supporting Team Coordination on the Ground: Requirements from a Mixed Reality Game
}

\author{
Joel E. Fischer, Wenchao Jiang, Andruid Kerne*, Chris Greenhalgh, \\ Sarvapali D. Ramchurn ${ }^{+}$, Steven Reece ${ }^{\sim}$, Nadia Pantidi and Tom Rodden \\ The Mixed Reality Lab, University of Nottingham, NG8 1BB, United Kingdom. \\ *Interface Ecology Lab, Texas A\&M University, College Station, USA. \\ Agents, Interaction and Complexity Group, University of Southampton, SO17 1BJ, UK. \\ Pattern Analysis \& Machine Learning, University of Oxford, OX1 3PJ, UK. \\ \{jef,wxj,cmg,nxk,tdr\}@cs.nott.ac.uk, andruid@ecologylab.net, sdr1@soton.ac.uk, \\ reece@robots.ox.ac.uk
}

\begin{abstract}
We generate requirements for time-critical distributed team support relevant for domains such as disaster response. We present the Radiation Response Game to investigate socio-technical issues regarding team coordination. Field responders in this mixed-reality game use smartphones to coordinate, via text messaging, GPS, and maps, with headquarters and each other. We conduct interaction analysis to examine field observations and log data, revealing how teams achieve local and remote coordination and maintain situational awareness. We uncover requirements that highlight the role of local coordination, decision-making resources, geospatial referencing and message handling.
\end{abstract}

\section{Introduction}

Highly coordinated, time-critical collaborative activities, such as disaster response (DR), have recently gained much attention of the collaborative systems community (e.g., Mendondça et al., 2007). Recent research initiatives at the boundary of Artificial Intelligence and Interactive Systems Design are proposing human-agent collectives (HACs) as a novel approach to designing systems to support such timecritical team coordination, where groups of humans and computational or embodied agents collaborate to achieve a common task ${ }^{1}$. Coordination is essential in such settings so that time critical interdependent activities such as search and rescue can be completed in a timely and satisfactory manner (Bradshaw, 2011). The critical nature of the DR domain makes it challenging to design and deploy HAC systems 'in the wild'. On the other hand, computational simulations of such scenarios are not only difficult to construct, but the veracity of results may be impossible to verify (Simonovic, 2009). In turn, little is known about the design space for HAC

\footnotetext{
${ }^{1}$ http://www.orchid.ac.uk/
}

(C) Springer 2014. This is the author's version of the work. It is posted here for your personal use. Not for redistribution. The definitive version was published at COOP 2014, May 27-30, 2014, Nice, France. 
systems to support time critical coordination settings such as DR, for example to help responders with spatial task prioritization (Ramchurn et al., 2010).

What are the challenges and requirements in building systems to support team coordination in such settings? Before we introduce 'smart' agent systems, we need to understand how teams coordinate in time critical settings using communication and situation awareness tools. In order to explore this design space and to generate requirements for technologies to support team coordination, we developed the Radiation Response Game. We adopt a serious mixed-reality games approach to create a setting in which participants experience physical exertion and stress through bodily activity and time pressure, mirroring aspects of a real disaster setting (PAHO, 2001). We use game probes as a complementary approach to gathering system requirements for real-world settings, for example in addition to codesigning with users. Our game probe explores a socio-technical setting in which field responders receive guidance from a central command headquarters ('HQ'), inspired by the concept of the Sector Coordinator in USAR task forces (INSARAG, 2012). Participants collaborate to save spatially distributed 'targets' in an area affected by a spreading 'radioactive cloud', both locally, with collocated 'field responders', as well as remotely, with HQ and with distant field responders. Based on interaction analysis of team coordination in the game we generate requirements for technologies to support team coordination in time critical settings.

\section{Related Work}

We review disaster simulations and games that underpin our mixed-reality games approach, and work on team coordination that underpins our methodology.

Disaster Simulations and Games. Computational simulations, particularly agentbased simulations of disasters, are the predominant approach in the computing literature to predict the consequences of "courses of action" (Hawe et al., 2012), e.g., to model first responder information flow (Robinson and Brown, 2005), or logistic distribution of emergency relief supplies (Lee et al., 2007).

Limitations of the veracity of computational simulations are manifold. For example, Simonovic highlights that simulations may rely on unrealistic geographical topography, and most importantly, may not account for "human psychosocial characteristics and individual movement, and (...) learning ability" (Simonovic, 2009: 89). The impact of emotional and physical responses likely in a disaster, such as stress, fear, exertion or panic (Drury et al., 2009) remains underaddressed in approaches that rely purely on computational simulation.

One of our work's main objectives is to study interaction and coordination situated in rich and 'messy' real-world socio-technical settings. As it is difficult to deploy technological prototypes in real disasters, game-like simulations have been adopted to study technology interaction in disaster scenarios, for example to prepare first responders for scenarios in which hazardous materials are involved (Losh, 2007). Abbasi et al. (2012) present a study in which locally distributed participants played the role of victims asking for help via social media in a simulated 
crisis, and participants that played the role of first responders used a coordination system to filter messages and mobilize the appropriate responder teams according to their assigned capabilities. Toups, Kerne and Hamilton (2011) present the design and evaluation of the Team Coordination Game, which teaches participants effective cooperation and - in particular - communication, based on a zero-fidelity simulation of team coordination that focuses on distributed cognition in lieu of concrete details, yet draws directly from fire emergency response work practice.

We adopt a serious-mixed reality games approach (Fischer et al., 2012) to create a game probe that enables studying team coordination, interaction and communication in a real-world disaster scenario whilst providing confidence in the efficacy of behavioural observations. Suspension of disbelief occurs frequently in the play of pervasive or mixed-reality games (Stenros et al., 2009). Mixed-reality games bridge the physical and the digital (Benford et al., 2005). They serve as a vehicle to study distributed interactions across multiple devices and ubiquitous computing environments 'in the wild' (Crabtree et al., 2006).

Team Coordination. Malone (1990: 361) defines coordination as "the act of managing interdependencies between activities performed to achieve a goal".

In disaster response, team coordination is essential in order that groups of people can carry out interdependent activities together in a timely and satisfactory manner (cf. Bradshaw et al., 2011). Disaster response experts report that "failures in team coordination are the most significant factor in critical emergency response" (Toups et al., 2011:2) that can cost human lives. Shared understanding, situation awareness, and alignment of cooperative action through on-going communication are key requirements to enable successful coordination. Convertino et al. (2011) design and study a set of tools to support common ground and awareness in emergency management. For our game probe, we study how participants coordinate teams and perform spatially distributed, time critical tasks.

One important characteristic of large-scale disaster is the presence of multiple spatially distributed incidents (Chen et al., 2005). To deal with multiple incidents, the disaster response team has to coordinate spatially distributed resources and personnel to carry out operations (e.g. search, rescue and evacuation).

Depending on the proliferation of incidents, response personnel may need to dispatch, deploy and redeploy limited resources. Coordination is required to efficiently allocate limited resources to multiple incidents with temporal and spatial constraints imposed by the nature of disasters.

Mixed-reality games (MRG) share a common set of characteristics with time critical settings, such as disaster response (DR):

- Bridging the physical and the digital. Both DR as well as MRGs routinely bridge the physical and the digital as part of their actors' coordination (Benford et al., 2005). DR for example makes use of the twitterverse to inform real world response (e.g., Sarcevic et al., 2012).

- Orchestration. DR and MRGs are both highly orchestrated activities. Authoring and orchestration tools 'behind the scenes' of an MRG, as well as player interfaces, provide managers, players and spectators with different temporal and spatial views of the game world in order to support the experience (Crabtree et 
al., 2004). These settings are surprisingly comparable to the 'control room' of a disaster response operation, in their collections of sophisticated technological arrangements to communicate and coordinate real-time information streams, in order to create a holistic view amidst an immersive setting of interest.

- On-the-ground and online. In both DR, as well as in MRGs, people on the ground often work with people online to solve a common problem. Sarcevic et al. (2012) show how understanding online content can foster understanding of medical coordination challenges in DR on the ground. MRGs often leverage the fact that people on the ground and online have different views of the world, which are turned into different abilities within the game (Flintham et al., 2003).

These key characteristics illustrate the overlap between time-critical coordination in MRGs and DR. This perspective underlies our motivation to explore the approach of studying team coordination through a game probe.

To assess team coordination and performance, Borge et al (2012) analyse interaction, communication and tasks to identify primary team activities. Convertino et al. (2011) analyse the turn-taking structure of communication and dialog acts (speech acts) to assess how participating teams use situational awareness tools.

We draw on interaction analysis (Jordan and Henderson, 1995) and message classification based on speech act theory (Searle, 1975) to assess participants' team coordination in the game probe. Methods are detailed further below.

\section{The Radiation Response Game}

We designed and implemented the Radiation Response Game in order to study team coordination through a location-based, mixed-reality game probe. In the following sections, we describe the game, including grounding the design rationale, game interfaces, iterative design process, and the system architecture.

Design Rationale. The Radiation Response Game is based on the fictitious scenario of radioactive explosions that create expanding and moving radioactive clouds, which pose a threat to responders on the ground (field responders), and (virtual) targets to be rescued from around the game area. We chose a radiation scenario because unlike disasters that cause physical devastation, radiation poses an 'invisible threat', which creates the need to monitor the environment closely with sensing devices, and to communicate frequently.

Field responders are supported by a centrally located 'headquarters' (HQ) control room, staffed by coordinators. Players exchange messages through an instant messaging style communication system. Messages are broadcast, which means they are visible to all players. While formal response teams tend to use radio to communicate (e.g., Toups et al., 2011), we chose text-based messages for its flexibility to support scenarios with many distributed field responders. Text messaging is a realistic option for increasing the throughput of coordinators severely constrained by time and workload. 
We designed core game mechanics to provoke exploration of specific aspects of team coordination. The game mechanics are motivated by real world challenges of resource and task allocation for coordinating spatially distributed resources and personnel to carry out operations (Chen et al., 2005).

The game's two-tiered organisational structure is derived from real world disaster response organisation and from NIMS (Homeland Security, 2008). The game's HQ is loosely modelled on sector coordinators, whose role is to manage resources and communications between their assigned teams, and command and coordinate action within their sector (INSARAG, 2012). Field responders are modelled on team leaders and members. We ignore this distinction to simplify roles, assignments, and game mechanics.

Responder roles and targets. Each field responder is assigned one of four roles:<smiles>CC(C)(C)Cc1ccccc1</smiles>

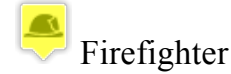
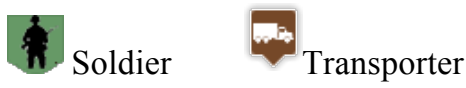

There are four types of (virtual) targets:

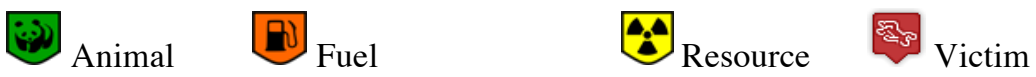

The objective of the field responders is to rescue as many targets as possible by 'carrying' them to a drop off zone. To pick up and carry one of the target objects, two responders with particular appropriate roles are required in immediate proximity to the object. For example, a soldier and a transporter are required to pick up and carry fuel, and a medic and a soldier are required to pick up an animal.

The role-target mapping mechanic requires players to engage in resource coordination. Field responders have to engage in 'agile teaming' - forming, disbanding, relocating and re-forming in teams over the course of the game in order to complete the game objective. This is an example of what Toups et al call, information distribution (2011).

The radioactive cloud. The "cloud" is a danger zone that can incapacitate field responders. It imposes spatial and temporal constraints on task performance and health levels. The cloud is analogous to various spatial phenomena in disasters (e.g. spreading fires, diseases and floods). In order to incentivise communication between HQ and field responders, the spatial position and movement of the cloud is only known to HQ.

Command-and-control structure. The division of responsibility into HQ and field responders simulates a situation where volunteer responders are connected to a simple two level Command-and-control structure, similar to the real-time layer of the existing professional disaster response organizations (e.g., Chen et al., 2005).

Coordination interfaces. Field responders are equipped with a 'Mobile Responder App' providing them with sensing and awareness capabilities (see figure 1). The app shows a measure of radioactivity (i.e., using a Geiger counter), their 'health level' based on radioactive exposure, and a GPS-enabled map of the game 
area with the targets to be collected and the drop off zones for the targets. Icons according to responder roles that additionally have their initials on them can be used to identify individuals. Another tab reveals the messaging widget to broadcast messages to the other field responders, and to headquarters.

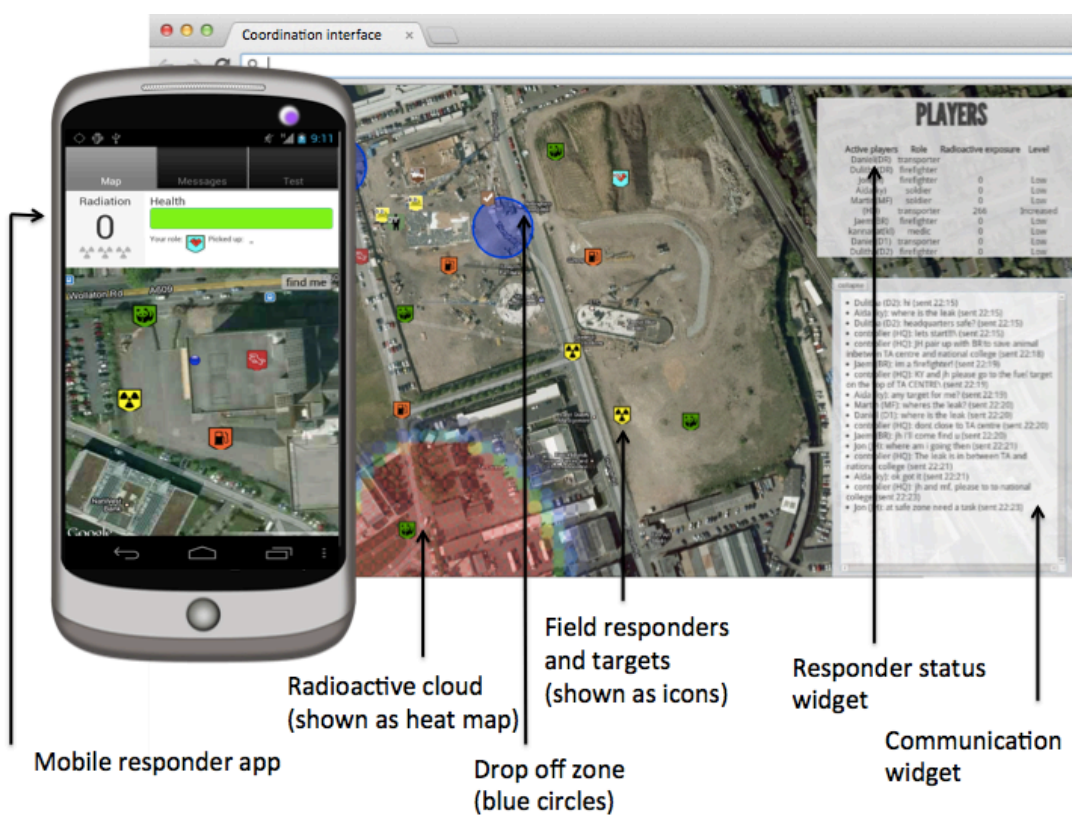

Fig. 1. Field responders and HQ coordinators have complimentary views of the game terrain (Mobile Responder App and HQ Coordination Interface).

HQ is operated by at least two coordinators. They access a browser-based 'coordination interface' that provides an overview of the game area, including real-time information of the players' locations (see figure 1). HQ can also broadcast messages to all field responders, and can review the responders' exposure and health levels. Importantly, only headquarters has a view of the radioactive cloud. 'Hotter' zones correspond to higher levels of radioactivity.

Iterative design. We briefly describe three cycles of iterative game design and evaluation.

In the first iteration, we used a paper-based prototype to test and refine the core game mechanics. We recruited 12 participants, allocated one of four roles to them, and equipped them only with paper maps with locations of targets. They had to form different kinds of teams to retrieve the different kinds of boxes placed in the game area. The paper prototype demonstrated the demand for better support of situation awareness and communication to enable coordination.

The technology prototype was first tested with users in the second iteration. Users were equipped with the responder smartphone app to communicate, navigate, locate and pick up targets in teams formed according to role requirements. HQ was staffed by members of the research team. A pilot study was conducted 
with members of the public that visited an Open Day at a local university. A total of 20 members of the public tested the game in four ad-hoc game trials. The lessons learned in the pilot study revealed problems with user interaction, networking, and game parameter tuning, which we subsequently addressed.

In the third iteration, we improved system stability and interface designs. We conducted a pilot study at the campus of another university, to test the system in place. The full-fledged study we report on here was conducted shortly thereafter.

System architecture. The Radiation Response Game is based on the opensourced geo-fencing Map-Attack ${ }^{2}$ game platform, which has been iteratively developed to provide responsive, (relatively) scalable experiences. Our mixed-reality game relies especially on real-time data streaming between client and server. Client-side requests for less dynamic content use HTTP. Frequent events, such as location updates and radiation exposure, are streamed to clients to avoid the overhead of HTTP. In this way, field responders are kept informed in near real-time.

The platform is built using web technologies such as socket.io, node.js, Ruby Sinatra, and the Google Maps API. We built on the existing open source MapAttack app for Android to develop the Mobile Responder App.

\section{Study Design}

To explore socio-technical issues around team coordination, we ran two Radiation Response Game sessions, with volunteers recruited from the local university. We describe participants, procedure, session configuration, and methods used to collect and analyse quantitative and qualitative data.

Participants. Study participants were recruited through posters and emails. A total of 18 participants were recruited ( 8 female); 7 participated in session $\mathrm{A}$ and 11 in session B. All participants were reimbursed with 15 pounds for 1.5 hours of study. The majority of participants were students of the local university.

Procedure. Upon arrival in the HQ (set up in a meeting room at the local university), participants were briefed and asked to consent to participate. Roles were randomly assigned to all participants (HQ/field responders: firefighter, medic, transporter, soldier). Field responders were provided with a smartphone; HQ coordinators with a laptop. Game rules and interfaces were introduced, and participants were assisted in setting up their phones and laptop clients. Field responders and HQ coordinators were given 5 minutes to discuss a common game strategy. All field responders were accompanied to the starting point within the designated game area, about 1 minute walk from headquarters.

Once field responders were ready to start, HQ sent a "game start" message. Gameplay commenced for 30 minutes. A "Game over" message by HQ concluded the game. Field responders returned to HQ for the post-game session.

The post-game session consisted of a questionnaire aimed at collecting participants' feedback on (1) first impressions of the game; (2) usability of the system,

\footnotetext{
${ }^{2}$ http://mapattack.org/
} 
and; (3) coordination issues in the game. A group interview was then conducted, before participants were debriefed and dismissed.

Game configuration. The size of the game area on the local university campus was 400 by 400 meters, without heavy traffic. The terrain of the game area includes grassland, a lake, buildings, roads, and footpaths and lawns. There are two drop off zones and 16 targets. The pilot study showed that this was a challenging, yet not too overwhelming number of targets to collect in a 30 min game session. There were four targets for each of the four target types. The pattern of cloud movement and expansion was the same for both game sessions.

Methods. We took a mixed methods approach to data collection and analysis. In addition to quantitative questionnaires, a semi-structured group interview was conducted aimed at eliciting important decision points, strategies and the overall decision-making process. Furthermore, five researchers with camcorders recorded the game play. One researcher recorded action in the HQ, and four other researchers each recorded a field responder team.

We developed a log file replay tool to help with data analysis of time stamped system $\operatorname{logs}$ that contain a complete record of the game play, including responders' GPS location, their health status and radioactive exposure, messages, cloud location, locations of target objects and task status.

Interaction analysis of local coordination. We focus on the analysis of local field responders' interaction to unpack team coordination, including handling of messages sent by HQ. Video recordings of field action were catalogued to identify sequences (episodes) of interest (cf. Heath et al., 2010). Key decision points in teaming and task allocation served to index the episodes. Interesting distinct units of interaction were transcribed and triangulated with log files of relevant game activity for deeper analysis that we present in this paper.

Message classification. How are remote messages used as a coordination resource? We used speech-act theory and the notion of adjacency pairs in linguistics to classify messages sent between and among responders and HQ.

According to speech act theory, utterances in dialogues can be considered as speech acts from three dimensions. We were primarily concerned with the illocutionary dimension of speech acts. Searle's classification of illocutionary acts (Searle, 1975) is used to categorize messages in the communication system.

\section{Results}

Here, we present findings from interaction analysis supported by message classification that reveal how team coordination was achieved. Overall, responders rescued 7 and 9 targets in session A and B respectively, out of 16 targets in total per session. Two players were incapacitated in session A, and 1 player was incapacitated in session B. 117 and 70 messages were sent in session A and B, respectively. We used Searle's classification of speech acts to categorize messages (see table 1). We also add requests to the table to categorize all of the messages (Searle does not classify those as speech acts). 


\begin{tabular}{|c|c|c|c|c|c|c|}
\hline \multirow[t]{2}{*}{ Speech acts } & \multicolumn{2}{|c|}{ Session A } & \multicolumn{2}{|c|}{ Session B } & \multirow[t]{2}{*}{ Example } & \multirow[t]{2}{*}{ Total } \\
\hline & HQ & FR & HQ & FR & & \\
\hline Directives & 57 & 0 & 32 & 0 & $\begin{array}{l}\text { JH pair up with BR to save animal in be- } \\
\text { tween TA centre and national college. }\end{array}$ & $89(47 \%)$ \\
\hline Assertives & 25 & 2 & 8 & 4 & The leak around geospatial is bigger. & $39(20 \%)$ \\
\hline Expressives & 5 & 0 & 0 & 0 & Good Job, JJ, TV and RL. & $5(2 \%)$ \\
\hline Declarations & 3 & 0 & 0 & 0 & NOTICE - TEAM B: NS + TD. & $3(1.6 \%)$ \\
\hline Commissives & 0 & 4 & 0 & 4 & ok got it. & $8(4 \%)$ \\
\hline Requests & 8 & 6 & 1 & 19 & wheres the leak? & $34(18 \%)$ \\
\hline Unclassified & & & & & & $9(5 \%)$ \\
\hline
\end{tabular}

Table 1. Classification of messages (based on speech act theory).

We present four episodes to illustrate team coordination. By example of the first episode, we unpack how field responders account for messages from HQ, in particular issues with how directives on task allocation and execution are addressed. An overview shows that directives from HQ are frequently not brought up locally. A further episode demonstrates how field responders instead draw on technological and embodied resources to achieve local coordination, without HQ involvement. Finally, two more examples illustrate how responders routinely employ messages as a resource to support situational awareness.

Responding to directives from HQ. We examine how field responders deal with messages from HQ that attempt to allocate tasks and manage task execution (i.e., directives). Classification of messages showed that directives were exclusively sent by HQ, and that they were the most frequent kind of message (Table 1). Directives index (attempted) instances of remote coordination of field responders by HQ. The observed response to messages is critical to understanding relationships between local and remote coordination. The following episode depicts a team of three on their way to pick up fuel. Their path is blocked by radiation. Without a team, firefighter $\mathrm{JH}$ (on the left) has just joined soldier KY (on the right), and firefighter D2 who have just been allocated a task in a message by HQ. 


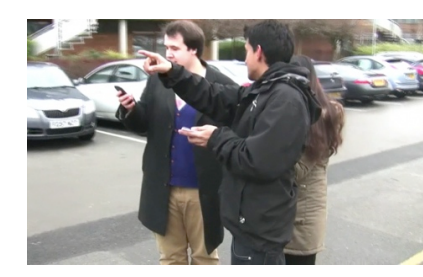

KY: ((reading out message)) KY and D2, please walk fast to the junction and quickly return back ((laughs)) D2: Oh is that what we have to do? ok so we have to run to $(2.0)$ We need to work out where we have to run to first and then get (.) get it back. Which junction is that? If you run to the next (0.5) thing ( (points)), and then come back $\overline{(1.0)}$ that would work $(1.0)$ is it safer to go around?

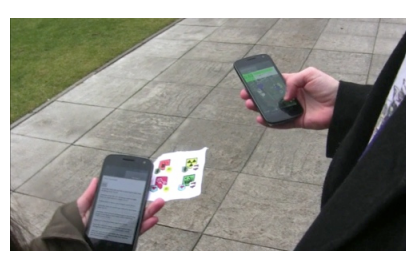

[The team tries to go around the cloud but is stopped by radiation, realising their target is in the cloud. Meanwhile, D2 has left due to increased exposure.]

KY: So we have to run! [through the radiation]

JH: Do we have to run through the (.)

through the radiation? ((looking at map)) $\mathbf{K Y}:$ Yah this is what the headquarters told us to do ((looking at messages))

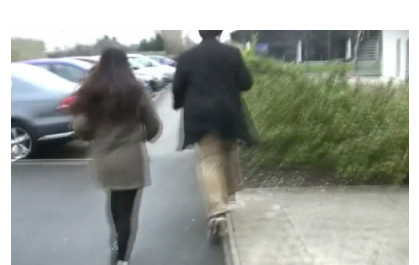

H: I have a terrible feeling thats gonna $\mathrm{kill}$ us.

KY: But its gonna be meaningful

( ( laughs))

JH: We go around this corner, if it gets to half [referring to health] we should probably start running back.

[KY JH begin running into the cloud]

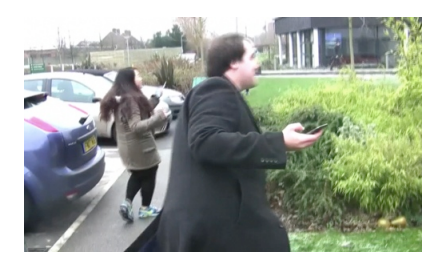

KY : ( (yells)) OH OH! It's a hundred! [refers to radiation level]

JH: We are basically in the middle of it! We are basically in the middle of it!

KY: ((shouts)) I'm going back! Get the fuel first! Get the fuel first! oh no! JH: We are not prepared for that! I blame our HQ.

[They turn around and run back out of the cloud without the fuel.]

This episode begins with a message by HQ attempting to help give directions to the target. D2's response to the message is hesitant (is that what we should do?). His following question (which junction is that?) suggests the referent in HQ's message is not understood. They attempt to go around the radiation. They realise their target is in the cloud. They refer back to the message to support their intent to go into the cloud to attempt to save the target (Yah this is what the headquarters told us to do). Having run into the cloud, they refer to the Geiger counter and realise the exposure is too high. Meanwhile, their health is decreasing rapidly. They abandon the task and flee to safety, whilst JH expresses his frustration (We are not prepared for that. I blame our HQ.).

First, the episode shows that geospatial referencing in messages can be problematic. It is unclear to the responders which junction HQ is referencing (and the 
responders do not ask for clarification), so they revise the route themselves. At the same time, they draw on the messages to justify their entering of the cloud. It does not occur to the responders that HQ allocated the task at an earlier time, before the cloud had covered the target. HQ does not update the responders on the increased danger, or revise their earlier task allocation. When the responder team fails to complete the task, they place blame instead of thinking self-critically.

Overview: how field responders addressed task allocation messages. Overall, out of the 43 task allocation directives HQ sent, the recipient field responders brought up only 15 messages in conversation in the team. The instances in which task allocation messages were addressed reveal the handling and value of HQ directives in the local coordination. Firstly, out of the 15 task allocation messages responders talked about, they decided to ignore the instructions only once. The responders ignored instructions because they were engaged in another task that they did not want to abandon. Secondly, four HQ instructions to rescue a certain target coincided with the same plan that had already been made locally by the responders. In 10 cases, field responders chose to follow the instructions. However, due to confusion and misunderstanding they failed to follow them correctly six times. In fact, only 2 instances of directives from the HQ led to task completion. For the remaining 14 saved targets, field responders had locally allocated the tasks without HQ.

Local coordination without HQ. As presented, field responders predominantly coordinated teaming and task allocation of targets that were saved without HQ involvement. The following episode illustrates how field responders achieve coordination of teaming and task allocation locally. We join the action as BR and another responder are waiting at the drop-off zone without a compatible teammate, as $\mathrm{MF}$ and his teammate join and drop-off their target.

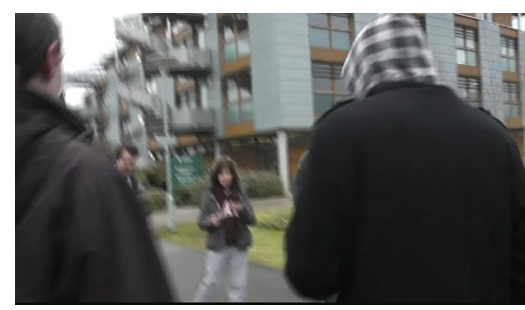

[MF (on the right) and teammate walking towards BR (center)]

BR: Any soldiers?

MF : I am soldier yeah.

BR: Would you like to pair with me?

$(2.0)$ to rescue a fuel

MF : what are you after?

BR: I am a firefighter.

MF: Soldier and firefighter is fuel

isn't it?

$\mathrm{BR}$ : yeah.

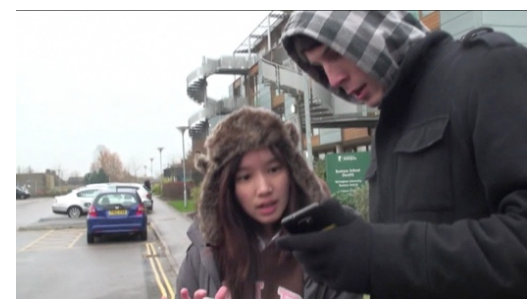

MF : What can we get? (2.0) ((looks

at screen)) this one in the center? ((points at screen))

BR: ((glances MF's screen)) I think

there are two people going for

that. I think we should go for this

one ((points at screen)).

MF : We are going to get killed

( (both laugh $))$.

[The team begins walking to target.] 
The episode shows how teaming and task allocation are achieved opportunistically between BR and MF, with BR already waiting at the drop-off zone. Field responders also confirmed their opportunistic behaviour in the interview:

"Just save the closest target then just pair up and go to the other one" [P2]

"We just check, with that group, which target we can get. We see on the map to find the closet one we can get." [P4]

BR and MF can then be observed sharing the screen of his device and using the map to identify potential targets. They realise one of them is already being pursued by another team. They agree on another target to pursue. Note that messages do not play a role in this episode. It exemplifies how teaming and task allocation are achieved locally, without consulting HQ.

Messages as a resource of situational awareness. In the Radiation Response Game, field responders need to be aware of what other responders are doing, where the 'danger zone' is (the cloud), and where it is likely to move. Awareness of each other's actions helps responders avoid conflicts in planning, while awareness of the danger zone is essential to survive. The following episode illustrates how responders use messages as a resource to gain situational awareness.

The episode takes place towards the end of game session B. The radioactive cloud has grown so much that navigation in the game area becomes increasingly difficult. MF is with a group of five responders, two of which are carrying an animal. The cloud is blocking their way towards the drop off zone; they stop.

MF: ((reads message from Ho out loud)) There is another leak around Geospatial. (1.0) Which is Ah: so there's a leak sprung up there. ((points)) Geospatial is like (.) that building right there. They say there is another leak. We should go all the way round (0.5) to the top left one, I think.

MF brings up HQ's message of the new leak, and suggests a route around the new cloud. The group ends up following MF's route suggestion as a result. News of the new cloud, provided by HQ, enables the group to change their route to avoid danger. We commonly observed responders sharing information that provides situational awareness through face-to-face conversation. In the previous example, MF shared the message with a group of responders he was with already. The following example takes place between D2 and his teammate, as they are approached by $\mathrm{JH}$, who is currently without teammate.

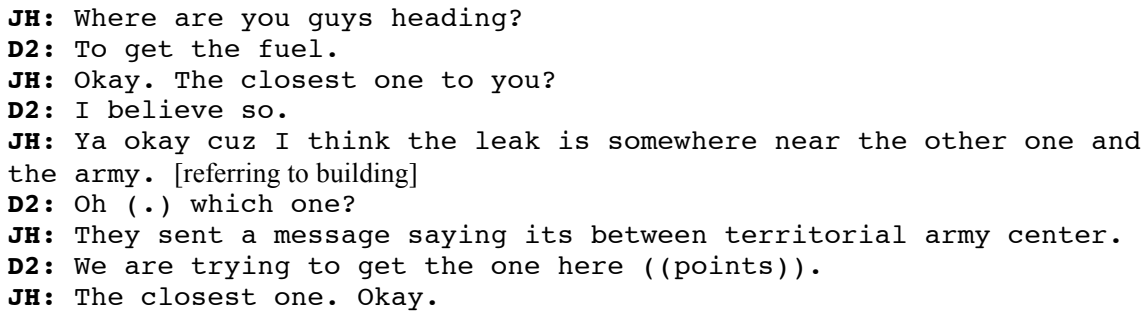


Making use of the map as he approaches them, JH asks the others to clarify which fuel they intend to pursue (the closest one to you?). He proceeds to inform the team that the "leak is somewhere near the other one". D2's response (Oh, which one?) suggests they did not know this. In turn, JH elaborates on the location of the cloud, using an anonymous "they" to refer to the source of his information. "They" is likely to refer to HQ as they previously sent a message with the information of the cloud's location. Conversational sharing of important information was a common resource responders employed to achieve and maintain situational awareness. However, requests for information were regularly not reciprocated with a response: out of 14 requests in session A, 8 were not responded upon; and in session B, 14 out of 20 requests were not responded upon.

\section{Requirements for Team Coordination Support}

We now discuss the requirements for team coordination that emerged from the game and relate them to broader concerns for the design of HAC systems that support team coordination.

The embodied game probe embedded responders in a challenging setting. They needed to communicate effectively to make time critical decisions on teaming and task allocation, both locally in the field as well as remotely through messaging. Field responders physically engage and navigate the environment to perform tasks while maintaining awareness of risk and danger. The data reveals multiple challenges for team coordination involving communication and decision-making.

Local decision-making. The study showed that teaming and task allocation were predominantly organised locally among field responders, in an opportunistic, onthe-fly fashion. Despite the fact that headquarters attempted to coordinate task allocation remotely, few of these directives were brought to conversation locally. Only 2 out of 16 tasks that field responders completed were remotely allocated by HQ. Local decision-making needs to be supported by HAC systems that aim to support time-critical team coordination, and need to integrate capabilities to enable team-wide sharing of the local decisions.

Coordinate resources. While field responders made decisions on teaming and task allocation in a seemingly ad hoc fashion, game data reveals how field responders draw on resources to achieve situational awareness in order to coordinate successfully. A common understanding of the location and movement of the radiation cloud was achieved by sharing information from game messages verbally in a local group. Face-to-face talk was an essential resource for relaying information from the Mobile Responder App to teammates, such as radioactive exposure, others' whereabouts, task status, and other monitoring of the broadcast messages. Future HAC systems need to take into account that such coordinate resources are likely to be comprised of digital as well as embodied human resources.

Geospatial referencing. The results show that geospatial referencing was problematic in various ways, particularly in directive messages sent to the field play- 
ers. Participants had different levels of knowledge of the campus, which made understanding of landmark references uncertain. Some participants also struggled with making sense of north/south/east/west directions in relation to their current position and orientation. To deal with misunderstandings, players had to ask for clarification via messages or spend valuable time discussing the reference locally in order to understand it. Consistent with the findings of Toups et al. (2009), designers need to think carefully about how the presentation layer of HAC systems may be augmented with information that facilitates geospatial referencing (e.g., grids, labelling etc.) to facilitate human in addition to machine readability.

Freshness of messages. Problems arose from erroneous instructions or otherwise out-dated messages sent to field responders. In one case HQ sent a message in which two players with non-compatible roles were instructed to team up. This was particularly costly, as the players attempted to team up, and lost valuable time until they realised the game mechanics barred them from forming a team.

As demonstrated in one of the episodes, reading out-dated messages in a dynamically changing environment can contribute to responders taking dangerous actions that they believe to be safe, because they do not realise that the information is out-dated. However, in most cases, recipients managed to identify temporally irrelevant messages, and thus avoided following them.

To reduce confusion about message freshness, HAC systems should address these issues at the UI level, both for responders and for HQ. Develop functionality to flag messages as out-dated or retract incorrect messages. Up-to-date messages are particularly valuable. Thus, our findings support the use of fresh social media as a source of information for disaster response, despite problems that can arise with validation, because crowdsourced information will in many cases provide better coverage than official sources.

Acknowledgement of messages. In most cases, field responders did not acknowledge or respond to messages sent by the HQ. This was particularly problematic for directives (task allocation), as task status and field responder compliance often had to be inferred by observing their location updates on the map. This consumed HQ attention, with negative impact on HQ's overall work on state assessment and task planning. Observations in the field suggest that the physical demands (e.g., co-located team movement through terrain at speed) and cognitive demands to maintain situational awareness (e.g., monitoring of radioactivity and messages) are likely factors that explain lack of acknowledgement.

As a result, user interfaces that enable and encourage field responders to quickly acknowledge HQ messages, with minimum cognitive load, should be considered for messaging in HAC systems in such high demand settings. For effective team coordination in disaster response, interface and workflow designs need to factor in cognitive load and task demands for effective information distribution. 


\section{Conclusions}

The objective of the research presented here was to generate requirements for supporting time-critical team coordination. In particular, we focussed on a scenario in which responders coordinate role-based teaming and spatially distributed task allocation and execution using a real-time location and messaging system.

We presented the design and study of the Radiation Response Game as a mixed-reality game probe to investigate challenges for team coordination in a setting in which participants experience both physical strain through bodily activity, and cognitive challenge through time pressure and task complexity. We eschew high-fidelity simulations in favour of mixed-reality game probes as a platform for investigation of concomitant socio-technical issues: handling of mobile devices to communicate and maintain situational awareness (messaging, sensing, interaction, and display) intersect with face-to-face interaction, whilst the physio-cognitive challenges created through game mechanics and environment induce stress. We created a setting that allows exploring requirements to support team coordination of relevance to time-critical coordination domains such as real disaster response.

Findings from interaction analysis of field observations, triangulated with log files, reveal how field responders achieved coordination by drawing on local faceto-face conversation with fellow responders, and situational information provided by the interactive map, the Geiger counter, and the messages sent by HQ. Drawing on these findings, we generated requirements for supporting team coordination, emphasising the roles of local coordination, decision-making resources, geospatial referencing and message handling. These requirements inform future work on building human-centred HAC systems by emphasising the role of human interaction in team coordination in time-critical settings.

Acknowledgments. We are grateful for the support by the EPSRC ORCHID project (EP/I011587/1). Thanks to the University of Nottingham's Horizon Digital Economy Research Institute for support provided by a sabbatical grant to the third author.

\section{References}

Abbasi, M.-A., Kumar, S., Filho, J. A. A., \& Liu, H. (2012). Lessons learned in using social media for disaster relief - ASU crisis response game. In: Proc. of the 5th int. conf. on Social Comp., Behav.-Cultural Modeling and Prediction. Springer. 282-289.

Benford, S., Magerkurth, C., Ljungstrand, P.: Bridging the physical and digital in pervasive gaming. CACM 48(3) (March 2005), 54-57.

Borge, M., Ganoe, C. H., Shih, S.-I., \& Carroll, J. M. (2012). Patterns of team processes and breakdowns in information analysis tasks. In: Proc. CSCW '12. ACM Press.

Bradshaw, J.M., Feltovich, P.J., Johnson, M. (2011): Human-Agent interaction. In Boy, G.A. (ed.): The Handbook of Human-Machine Interaction: A Human-Centred Design Approach. Ashgate Publishing Company, Surrey, England. 283-299.

Chen, R., Sharman, R., Rao, H. R., \& Upadhyaya, S. J. (2005). Design Principles of Coordinated Multi-incident Emergency Response Systems. Simulation, 3495, 177-202. 
Convertino, G., Mentis, H. M., Slavkovic, A., Rosson, M. B., \& Carroll, J. M. (2011). Supporting common ground and awareness in emergency management planning. ToCHI, 18(4), 134. ACM Press.

Crabtree, A., Benford, S., Rodden, T., Greenhalgh, C., Flintham, M., Anastasi, R., Drozd, A., Adams, M., Row-Farr, J., Tandavanitj, N. and Steed, A. (2004). Orchestrating a mixed reality game 'on the ground'. In Proc. CHI '04. 391-398. ACM Press.

Crabtree, A., Benford, S., Greenhalgh, C., Tennent, P., Chalmers, M. and Brown, B. (2006). Supporting ethnographic studies of ubiquitous computing in the wild. In Proc. DIS '06. 6069. ACM Press.

Drury, J., Cocking, C., \& Reicher, S. (2009). Everyone for themselves? A comparative study of crowd solidarity among emergency survivors. The British journal of social psychology / the British Psychological Society, 48(3), 487-506.

Fischer, J.E., Flintham, M., Price, D., Goulding, J., Pantidi, N., Rodden, T. (2012). Serious mixed reality games. In: Mixed Reality games. Workshop at ACM CSCW 2012.

Flintham, M., Benford, S., Anastasi, R., Hemmings, T., Crabtree, A., Greenhalgh, C., Tandavanitj, N., Adams, M., Row-Farr, J. (2003): Where on-line meets on the streets: experiences with mobile mixed reality games. In: Proc. CHI '03.569-576. ACM Press.

Hawe, G. I., Coates, G., Wilson, D. T., \& Crouch, R. S. (2012). Agent-based simulation for large-scale emergency response. ACM Computing Surveys, 45(1), 1-51.

Heath, C., J. Hindmarsh, and P. Luff (2010): Video in qualitative research. Sage.

Homeland Security. (2008). National Incident Management System (NIMS).

INSARAG (International Search and Rescue Advisory Group). (2012). Assessment, Search and Coordination Methodologies. INSARAG Technical Note. United Nations.

Jordan, B. and Henderson, A. (1995). Interaction Analysis: Foundations and Practice. Journal of the Learning Sciences. Vol. 4, No. 1 (1995). 39-103. Taylor \& Francis.

Lee, Y. M., Ghosh, S., \& Ettl, M. (2009). Simulating distribution of emergency relief supplies for disaster response operations. In Proc. Winter Simulation Conference. 2797-2808.

Losh, E. (2007). The birth of the virtual clinic. Proceedings of the 2007 ACM SIGGRAPH symposium on Video games - Sandbox '07.73-80. ACM Press.

Malone, T. W., \& Crowston, K. (1990). What is coordination theory and how can it help design cooperative work systems? In Proc. CSCW '90. 357-370. ACM Press.

Mendonça, D., Jefferson, T. and Harrald, J. (2007). Collaborative adhocracies and mix-andmatch technologies in emergency management. Commun. ACM 50, 3, 44-49.

Pan American Health Organization. (2001). Stresss management in disasters. Washington, D.C.

Ramchurn, S., Polukarov, M., \& Farinelli, A. (2010). Coalition formation with spatial and temporal constraints. In Proc. AAMAS '10, IFAAMAS.

Robinson, C. D., \& Brown, D. E. (2005). First responder information flow simulation: a tool for technology assessment, 919-925.

Sarcevic, A., Palen, L., White, J., Starbird, K., Bagdouri, M., \& Anderson, K. (2012). "Beacons of hope" in decentralized coordination. In Proc. CSCW '12. 47-56. ACM Press.

Searle, John R. (1975). A Taxonomy of Illocutionary Acts. In: Günderson, K. (ed.), Language, Mind, and Knowledge. University of Minneapolis Press. 344-69.

Simonovic, S. P. (2009). Systems Approach to Management of Disasters: Methods and Applications. Disaster Prevention and Management (Vol. 20). Wiley.

Stenros, J., Montola, M., \& Waern, A. (2009). Pervasive game design strategies. Pervasive games: theories and design. Elsevier.

Toups, Z. O., Kerne, A., Hamilton, W. (2009). Game Design Principles for Engaging Cooperative Play: Core Mechanics and Interfaces for Non-Mimetic Simulation of Fire Emergency Response. In Proc. ACM SIGGRAPH Games 2009. ACM Press. 71-78.

Toups, Z. O., Kerne, A., \& Hamilton, W. A. (2011). The team coordination game. ACM Transactions on Computer-Human Interaction, 18(4), 1-37. 